\title{
A remark on resolution of terminal singularities
}

\author{
Yu. G. Prokhorov
}

\begin{abstract}
Let $(Z, o)$ be a three-dimensional terminal singularity of type $c A / r$. We prove that all exceptional divisors over $o$ with discrepancies $\leq 1$ are rational.
\end{abstract}

Let $(Z, o)$ be a three-dimensional terminal singularity of index $r \geq 1$ and let $\varphi: \tilde{Z} \rightarrow Z$ be a resolution. Let $S \subset \tilde{Z}$ be an exceptional divisor such that Center $(S)=o$. It is clear that the birational type of $S$ does not depend on $g$. By [1, 2.14] the surface $S$ is birationally ruled. We say that the corresponding discrete valuations $\nu=\nu_{S}$ of the function field $\mathcal{K}(Z)$ is rational (resp. birationally ruled) if so is the surface $S$. We are interested in the existence of rational exceptional divisors over $o \in Z$ with small discrepancies:

THEOREM. Let $(Z, o)$ be a three-dimensional terminal singularity of type $c A / r, r \geq 1$ and let $\nu$ be a divisorial discrete valuation of the function field $\mathcal{K}(Z)$ such that $a(\nu) \leq 1$ and $\operatorname{Center}_{Z}(\nu)=o$. Then $\nu$ is rational.

Recall that according to the classification [2], [3], $(Z, o)$ belongs to one of the following classes: $c A / r, c A x / 4, c A x / 2, c D / 2, c D / 3, c E / 2$, $c D, c E$. It was proved in the series of works [4], [5], [6] (see also [7], and $[\mathbf{8}]$ ) that for any $i \in \mathbb{N} \backslash r \mathbb{N}$ there exists an exceptional divisor $S$ with center at $o$ and discrepancy $a(S)=i / r$. Exceptional divisors with discrepancies $<1$ appear in any resolution. Divisors with discrepancy 1 and Center $=o$ appear in any divisorial resolution, i.e., in a resolution such that the exceptional set is of pure codimension 1 .

Proof of Theorem. Let $F \in\left|-K_{Z}\right|$ be a general member. Then $(F, o)$ is a Du Val singularity (of type $\left.A_{n}\right)$ [3, 6.4]. By the Inversion

The author was partially supported by the INTAS-OPEN 269 and Russian Foundation of Basic Research 99-01-01132. 
of Adjunction [9, 17.6] the pair $(Z, F)$ is plt. Let $q: Z^{q} \rightarrow Z$ be $\mathbb{Q}$ factorialization (see, e.g., [9, 6.7]). Then $Z^{q}$ has (terminal) singularities of types $c A / r_{i}$. Indeed, the surface $F^{q}:=q^{-1}(F)$ contains all singular points of $Z^{q}$ and $F^{q} \in\left|-K_{Z^{q}}\right|$. Since $F^{q} \rightarrow F$ is a crepant morphism, $F^{q}$ has only singularities of types $A_{n_{i}}$. Thus, replacing $Z$ with $Z^{q}$, we may assume that $Z$ is $\mathbb{Q}$-factorial.

Let $S$ be an exceptional divisor with center at $o$ and discrepancy $a(S) \leq 1$. Then $a(S, F)<1$. Since $K_{Z}+F$ is linearly trivial, we have $a(S, F) \leq 0$. Further, there is an 1-complement $K_{F}+\Theta$ of $K_{F}$ near $o$ (see [10, 5.2.3]). According to [11, 4.4.1] this complement can be extended to $Z$, i.e., there is an (integral) Cartier divisor $F^{\prime}$ such that $\left.F^{\prime}\right|_{F}=\Theta, K_{Z}+F+F^{\prime} \sim 0$, and $\left(Z, F+F^{\prime}\right)$ is lc. Then $a\left(S, F+F^{\prime}\right)=$ -1 . Now our theorem is a consequence of the following simple fact.

Proposition. Let $(Z, o)$ be a three-dimensional $\mathbb{Q}$-factorial terminal singularity and let $\nu$ be a divisorial discrete valuation of the field $\mathcal{K}(Z)$. Assume that there is a boundary $D$ such that the pair $(Z, D)$ is lc and $a(\nu, D)=-1$. Then

(i) The valuation $\nu$ is rational or is birationally a ruled surfaces over an elliptic curve;

(ii) if, moreover, $\lfloor D\rfloor$ there are at least two components passing through $o$, then $\nu$ is rational.

Proof. According to [9, 17.10] there is a blowup $f: X \rightarrow Z$ with irreducible exceptional divisor $S$ representing the valuation $\nu$ such that the log divisor $K_{X}+S+D_{X}=f^{*}\left(K_{Z}+D\right)$ is lc. Here $D_{X}$ is the proper transform of $D$. In this situation we have $\rho(X / Z)=1$. Therefore, $D_{X} \equiv-\left(K_{X}+S\right)$ is $f$-ample.

Consider a minimal log terminal modification $g: Y \rightarrow X$ of the pair $\left(X, S+D_{X}\right)$ (see, e.g., [11, 3.1.3]), i.e., a blowup such that $Y$ is $\mathbb{Q}$-factorial and

$$
K_{Y}+S_{Y}+D_{Y}+E=g^{*}\left(K_{X}+S+D_{X}\right),
$$

is dlt. Here $S_{Y}, D_{Y}$ are proper transforms of $S, D_{X}$, respectively, and $E=\sum E_{i}$ is a (reduced) exceptional divisor with $a\left(E_{i}, S+D_{X}\right)=-1$. Denote $\Delta:=\operatorname{Diff}_{S_{Y}}\left(D_{Y}+\sum E_{i}\right)$ and $\Omega:=\left.f^{*} D_{X}\right|_{S_{Y}}$. By [9, 17.7] the surface $S_{Y}$ is normal and the pair $\left(S_{Y}, \Delta\right)$ is lc. The assertion of (i) easily follows by the lemma below.

To prove (ii) we assume that $S_{Y}$ is not rational and $\lfloor D\rfloor$ is not irreducible. Then $S \cap\left\lfloor D_{X}\right\rfloor$ has at least two irreducible components. So is $S_{Y} \cap\left\lfloor D_{Y}+E\right\rfloor$. By the lemma below $\lfloor\Delta\rfloor$ has exactly two components (contained in $\left.\left\lfloor D_{Y}+E\right\rfloor\right)$ and the pair $\left(S_{Y}, \Delta\right)$ is plt. Further, $\left(S_{Y}, \Delta-\varepsilon \Omega\right)$ is klt whenever $0<\varepsilon$. For $0<\varepsilon \ll 1$, the pair $\left(S_{Y}, \Delta-\varepsilon \Omega\right)$ 
is a klt log del Pezzo (because $\Omega$ is nef and big). In this situation, $S_{Y}$ is rational (see, e.g., [11, 5.4.1]).

Lemma. Let $(S, \Delta)$ be a projective log surface with $\kappa(S)=-\infty$. Assume that $K_{X}+\Delta$ is lc and numerically trivial and the surface $S$ is nonrational. Then $S$ is birationally ruled over an elliptic curve and there exists at most two divisors with discrepancy $a(, \Delta)=-1$.

Proof (CF. [10, 6.9]). Replace $S$ with its minimal resolution and $\Delta$ with its crepant pull-back. There is a contraction $\phi: S \rightarrow C$ (with general fiber $\mathbb{P}^{1}$ ) onto a curve $C$ of genus $p_{a}(C) \geq 1$. In this situation pair $(S, \Delta)$ has only canonical singularities and all components of $\Delta$ are horizontal [11, 8.2.2-8.2.3]. Hence, divisors with discrepancy $a(, \Delta)=$ -1 are exactly components of $\lfloor\Delta\rfloor$. As an immediate consequence we have that the number of divisors with discrepancy -1 is at most two. If $\lfloor\Delta\rfloor \neq 0$, then for any component $\Delta_{i} \subset\lfloor\Delta\rfloor$ we have $2 p_{a}\left(\Delta_{i}\right)-2 \leq$ $\left(K_{S}+\Delta\right) \cdot \Delta_{i}=0$. Therefore, $p_{a}(C) \leq p_{a}\left(\Delta_{i}\right) \leq 1$. It remains to consider the case, when the pair $(S, \Delta)$ is klt. Again for any component $\Delta_{i} \subset \operatorname{Supp}(\Delta)$ we have $\Delta_{i}^{2} \leq 0$ (otherwise $\left(S, \Delta+\varepsilon \Delta_{i}\right)$ is a klt log del Pezzo). As above, $p_{a}(C) \leq p_{a}\left(\Delta_{i}\right)=\frac{1}{2}\left(K_{S}+\Delta_{i}\right) \cdot \Delta_{i}+1 \leq$ $\frac{1}{2}\left(K_{S}+\Delta\right) \cdot \Delta_{i}+1=1$.

Note that the assertion (ii) of our theorem is not true for other types of terminal singularities:

ExAmple (4]). Let $(Z, o)$ be a terminal $c A x / 2$-singularity $\left\{x^{2}+\right.$ $\left.y^{2}+z^{4 m}+t^{4 m}=0\right\} / \mathbb{Z}_{2}(0,1,1,1)$. Consider the weighted blowup with weight $\frac{1}{2}(2 m, 2 m+1,1,1)$. Then the exceptional divisor $S$ is given in $\mathbb{P}(2 m, 2 m+1,1,1)$ by the equation $x^{2}+z^{4 m}+t^{4 m}=0$. It is reduced, irreducible, and $a(S)=1 / 2$. It is easy to see that $S$ is a birationally ruled surface over a hyperelliptic curve of genus $2 m-1$.

\section{References}

[1] Reid M. Canonical threefolds, Géométrie Algébrique Angers, A. Beauville ed., Sijthoff and Noordhoff (1980) 273-310

[2] Mori S. On 3-dimensional terminal singularities, Nagoya Math. J. 98 (1985) 43-66

[3] Reid M. Young person's guide to canonical singularities, Proc. Symp. in Pure Math. 46 (1987) 343-416

[4] Kawamata Y. The minimal discrepancy coefficients of terminal singularities in dimension 3, appendix to 10$]$

[5] Markushevich D. Minimal discrepancy for a terminal CDV singularity is 1, J. Math. Sci. Univ. Tokyo 3 (1996) 445-456

[6] Shokurov V. V. Semistable 3-fold flips, Izv. Ross. Akad. Nauk Ser. Mat. 57 (1993) 165-222

[7] Shokurov V. V. 3-fold log models, J. Math. Sci., New York 81 (1996) 2667-2699 
[8] Hayakawa T. Blowing ups of 3-dimensional terminal singularities, Publ. RIMS, Kyoto Univ. 35 (1999) 515-570

[9] Kollár J. et al. Flips and abundance for algebraic threefolds, Astérisque. 211 (1992)

[10] Shokurov, V. V. Threefold log flips, Izv. Ross. Akad. Nauk Ser. Mat. 56 (1992) 105-203 (Russian); translation in Russian Acad. Sci. Izv. Math. 40 (1993) 95-202

[11] Prokhorov Yu. G. Lectures on complements on log surfaces, Mem. Japan. Math. Soc. 10, 2001

Department of Algebra, Faculty of Mathematics, Moscow State Lomonosov University, Moscow 117234, Russia

E-mail address: prokhoro@mech.math.msu.su 